\title{
$\Omega$ and $\Sigma^{0} \Lambda$ transition magnetic moment in QCD sum rules
}

\author{
Shi-lin Zhu \\ Department of Physics, Peking University, Beijing, 100871, China \\ and Institute of Theoretical Physics, Academia Sinica, P.O. Box 2735, Beijing 100080, China \\ W-Y. P. Hwang \\ Department of Physics, National Taiwan University, Taipei, Taiwan 10764 \\ Ze-sen Yang \\ Department of Physics, Peking University, Beijing, 100871, China \\ (Received 27 May 1997; published 8 January 1998)
}

\begin{abstract}
The method of QCD sum rules in the presence of the external electromagnetic $F_{\mu \nu}$ field is used to calculate the $\Omega$ magnetic moment $\mu_{\Omega}$ and the $\Sigma^{0} \Lambda$ transition magnetic moment $\mu_{\Sigma^{0} \Lambda}$, with the susceptibilities obtained previously from the study of octet baryon magnetic moments. The results $\mu_{\Omega}=-1.92 \mu_{N}$ and $\mu_{\Sigma{ }^{0} \Lambda}=1.5 \mu_{N}$ are in good agreement with the recent experimental data. [S0556-2821(98)03003-3]
\end{abstract}

PACS number(s): 13.40.Em, 11.55.Hx, 12.38.Lg, 14.20.Jn

According to the quark model the $\Omega^{-}$is composed of three strange quarks with parallel spins. This state is particularly interesting because of the large symmetry breaking that cannot be accommodated consistently in the naive quark model. In Ref. [1], it has been shown that nonstatic baryondependent magnetic effects are large. In this paper we investigate these symmetry breaking effects and evaluate the $\Omega$ moment in the QCD sum rule [3]. The $\Omega$ moment has been studied in literaures [2] and various theoretical results range from $-1.3 \mu_{N}$ to $-2.7 \mu_{N}$. We find our result is in good agreement with the recent data $[4,5]$.

In a typical hadronic scale the quantum chromodynamics (QCD) is highly nonperturbative which makes a direct analytical first-principles calculation impossible. In this work we adopt the method in the presence of an external electromagnetic field [6,7] to calculate the $\Omega$ and the $\Sigma^{0} \Lambda$ transition magnetic moment $\mu_{\Sigma^{0}{ }_{\Lambda}}$. The important aspect of this investigation is that the various susceptibilities in this calculation have already been determined from previous studies of octet baryon magnetic moments [6-9], and as a result our calculation is parameter free.

In the method of QCD sum rules [6,7], the two-point correlation function $\Pi(p)$ in the presence of an external electromagnetic field $F_{\alpha \beta}$ is written as

$$
\begin{aligned}
\Pi_{\Omega}(p) & =i \int d^{4} x\left\langle 0\left|T\left\{\eta_{\mu}(x), \bar{\eta}^{\mu}(0)\right\}\right| 0\right\rangle_{F_{\alpha \beta}} e^{i p \cdot x} \\
& =\Pi_{0}(p)+\Pi_{1}(p)(\sigma \cdot F \hat{p}+\hat{p} \sigma \cdot F)+\cdots, \\
\Pi_{\Sigma \Lambda}(p) & =i \int d^{4} x\left\langle 0\left|T\left\{\eta_{\Sigma^{0}}(x), \bar{\eta}_{\Lambda}(0)\right\}\right| 0\right\rangle_{F_{\alpha \beta}} e^{i p \cdot x} \\
& =\Pi_{2}(p)(\sigma \cdot F \hat{p}+\hat{p} \sigma \cdot F)+\cdots,
\end{aligned}
$$

where $\Pi_{0}(p)$ is the polarization operator without the external field $F_{\alpha \beta}$. The $\eta_{\mu}, \eta_{\Sigma^{0}}$, and $\eta_{\Lambda}$ are the currents with $\Omega$, $\Sigma^{0}$, and $\Lambda$ quantum numbers

$$
\eta_{\mu}(x)=\epsilon^{a b c}\left[s^{a T}(x) C \gamma_{\mu} s^{b}(x)\right] s^{c}(x),
$$

$$
\begin{aligned}
\eta_{\Sigma^{0}}(x)= & \epsilon^{a b c} \frac{1}{\sqrt{2}}\left\{\left[u^{a T}(x) C \gamma_{\mu} d^{b}(x)\right] \gamma_{5} \gamma^{\mu} s^{c}(x)\right. \\
& \left.+\left[d^{a T}(x) C \gamma_{\mu} u^{b}(x)\right] \gamma_{5} \gamma^{\mu} s^{c}(x)\right\}, \\
\eta_{\Lambda}(x)= & \epsilon^{a b c} \sqrt{\frac{2}{3}}\left\{\left[u^{a T}(x) C \gamma_{\mu} s^{b}(x)\right] \gamma_{5} \gamma^{\mu} d^{c}(x)\right. \\
& \left.-\left[d^{a T}(x) C \gamma_{\mu} s^{b}(x)\right] \gamma_{5} \gamma^{\mu} u^{c}(x)\right\},
\end{aligned}
$$

where $u^{a}(x), T$, and $C$ are the quark field, the transpose operators, and the charge conjugate operators. $a, b, c$ are the color indices. The interpolating currents couples to the baryon states with the overlap amplititude $\lambda$ :

$$
\begin{gathered}
\left\langle 0\left|\eta_{\mu}(0)\right| \Omega\right\rangle=\lambda_{\Omega} \nu_{\mu}(p), \\
\left\langle 0\left|\eta_{\Sigma^{0}}(0)\right| \Sigma\right\rangle=\lambda_{\Sigma} \nu_{\Sigma}(p), \\
\left\langle 0\left|\eta_{\Lambda}(0)\right| \Lambda\right\rangle=\lambda_{\Lambda} \nu_{\Lambda}(p),
\end{gathered}
$$

where $\nu_{\mu}$ is a vectorial spinor and satisfies $\left(\hat{p}-m_{\Omega}\right) \nu_{\mu}=0$, $\bar{\nu}_{\mu} \nu_{\mu}=-2 m_{\Omega}$, and $\gamma_{\mu} \nu^{\mu}=p_{\mu} \nu^{\mu}=0$ in the RaritaSchwinger formalism. $\nu(p)$ is a Dirac spinor.

On the hadronic level the correlators $\Pi_{1}(p)$ and $\Pi_{2}(p)$ are expressed in terms of the chirality-odd tensor structure $(\sigma \cdot F \hat{p}+\hat{p} \sigma \cdot F)$

$$
\begin{aligned}
\Pi_{1}(p)= & -\frac{1}{4} \mu_{\Omega} \frac{\lambda_{\Omega}^{2}}{\left(p^{2}-m_{\Omega}^{2}\right)^{2}}\left\{\frac{10}{9}+\frac{4}{9 m_{\Omega}^{2}}\left(p^{2}-m_{\Omega}^{2}\right)^{2}\right\} \\
& \times(\sigma \cdot F \hat{p}+\hat{p} \sigma \cdot F)+\cdots, \\
\Pi_{2}(p)= & -\frac{1}{4} \mu_{\Sigma 0} \frac{\lambda_{\Sigma} \lambda_{\Lambda}}{\left(p^{2}-\bar{m}^{2}\right)^{2}}(\sigma \cdot F \hat{p}+\hat{p} \sigma \cdot F)+\cdots,
\end{aligned}
$$

where $\{\cdots\}$ is 1 for the nucleon magnetic moment. The deviation from unity in Eq. (9) is due to the Rarita- 
Schwinger formalism for the spin $\frac{3}{2}$ field, of which the details may be found in Ref. [10]. $\bar{m}=\left(m_{\Sigma^{0}}+m_{\Lambda}\right) / 2$. We treat the $\Sigma$ and $\Lambda$ mass as degenerate due to their small mass difference since we never come across the poles and always work in the virtuality $p^{2}<0$. We denote the continuum and nondiagonal transition contributions simply by ellipses.

The external field $F_{\mu \nu}$ may induce changes in the physical vacuum and modify the propagation of quarks. Up to dimension six $(d \leqslant 6)$, we introduce three induced condensates,

$$
\begin{gathered}
\left\langle 0\left|\bar{q} \sigma_{\mu \nu} q\right| 0\right\rangle_{F_{\mu \nu}}=e_{q} \chi F_{\mu \nu}\langle 0|\bar{q} q| 0\rangle, \\
g_{s}\left\langle 0\left|\bar{q} \frac{\lambda^{n}}{2} G_{\mu \nu}^{n} q\right| 0\right\rangle_{F_{\mu \nu}}=e_{q} \kappa F_{\mu \nu}\langle 0|\bar{q} q| 0\rangle, \\
g_{s} \epsilon^{\mu \nu \lambda \sigma}\left\langle 0\left|\bar{q} \gamma_{5} \frac{\lambda^{n}}{2} G_{\lambda \sigma}^{n} q\right| 0\right\rangle_{F_{\mu \nu}}=i e_{q} \xi F^{\mu \nu}\langle 0|\bar{q} q| 0\rangle,
\end{gathered}
$$

where $q$ refers to $u, d$, and $s$ quark, and $e_{q}$ is the charge. The $\chi, \kappa$, and $\xi$ in Eq. (5) are the quark condensate susceptibilities and their values have been the subject of various studies. Ioffe and Smilga [6] found $\chi \approx-8 \mathrm{GeV}^{-2}$ with $\kappa=0$, $\xi=0$ in order to have $\mu_{p}=3.0 \mu_{N}$ and $\mu_{n}=-2.0 \mu_{N}$ $( \pm 10 \%)$. Balitsky and Yung [7] estimated

$$
\chi=-3.3 \mathrm{GeV}^{-2}, \quad \kappa=0.22, \quad \xi=-0.44
$$

using the one-pole approximation. Belyaev and Kogan [8] extended the calculation and obtained an improved estimate $\chi=-5.7 \mathrm{GeV}^{-2}$ using the two-pole approximation. Chiu et al. [9] also estimated the susceptibilities with the two-pole model and obtained

$$
\chi=-4.4 \quad \mathrm{GeV}^{-2}, \quad \kappa=0.4, \quad \xi=-0.8 .
$$

The values of these susceptibilities are consistent with one another except that the earliest result $\chi=-8 \mathrm{GeV}^{-2}$ in [6], is considerably larger (in magnitude) due to their neglect of $\kappa, \xi$ in the fitting procedure. In what follows, we shall adopt the condensate parameters $\chi=-4.5 \mathrm{GeV}^{-2}, \kappa=0.4, \xi=$ -0.8 which represent the average in the last three analyses.

The correlation functions $\Pi_{1}(p)$ and $\Pi_{2}(p)$ at the quark level are

$$
\begin{aligned}
\langle 0| & \eta_{\mu}(x) \bar{\eta}^{\mu}(0)|0\rangle_{F} \\
= & -2 i \epsilon^{a b c} \epsilon^{a^{\prime} b^{\prime} c^{\prime}}\left(\operatorname{Tr}\left\{S^{b b^{\prime}}(x) \gamma_{\mu} C\left[S^{a a^{\prime}}(x)\right]^{T} C \gamma_{\mu}\right\}\right. \\
\times & \left.S^{c c^{\prime}}(x)+2 S^{b b^{\prime}}(x) \gamma_{\mu} C\left[S^{a a^{\prime}}(x)\right]^{T} C \gamma_{\mu} S^{c c^{\prime}}(x)\right), \\
\langle 0| T & \eta_{\Sigma^{0}}(x) \bar{\eta}^{\Lambda}(0)|0\rangle_{F} \\
= & -\frac{2}{\sqrt{3}} i \epsilon^{a b c} \epsilon^{a^{\prime} b^{\prime} c^{\prime}}\left\{\gamma_{5} \gamma^{\mu} S_{s}^{a a^{\prime}}(x) \gamma_{\nu} C\left[S_{u}^{b b^{\prime}}(x)\right]^{T}\right. \\
& \times C \gamma_{\mu} S_{d}^{c c^{\prime}}(x) \gamma^{\nu} \gamma_{5} \\
- & \left.\gamma_{5} \gamma^{\mu} S_{s}^{a a^{\prime}}(x) \gamma_{\nu} C\left[S_{d}^{b b^{\prime}}(x)\right]^{T} C \gamma_{\mu} S_{u}^{c c^{\prime}}(x) \gamma^{\nu} \gamma_{5}\right\},
\end{aligned}
$$

where $i S^{a b}(x)$ is the quark propagator in the presence of the external eletromagnetic field $F_{\mu \nu}[6]$. We find

$$
\begin{aligned}
i S_{q}^{a b}(p)= & \delta^{a b} \frac{i}{\hat{p}-m_{q}}+\frac{i}{4} \frac{\lambda_{a b}^{n}}{2} g_{s} G_{\mu \nu}^{n} \frac{1}{\left(p^{2}-m_{q}^{2}\right)^{2}}\left[\sigma^{\mu \nu}\left(\hat{p}+m_{q}\right)+\left(\hat{p}+m_{q}\right) \sigma^{\mu \nu}\right]+\frac{i}{4} e_{q} \delta^{a b} F_{\mu \nu} \frac{1}{\left(p^{2}-m_{q}^{2}\right)^{2}} \\
& \times\left[\sigma^{\mu \nu}\left(\hat{p}+m_{q}\right)+\left(\hat{p}+m_{q}\right) \sigma^{\mu \nu}\right]-\delta^{a b} \frac{\langle\bar{q} q\rangle}{12}(2 \pi)^{4} \delta^{4}(p)-\delta^{a b} \frac{\left\langle g_{s} \bar{q} \sigma \cdot G q\right\rangle}{192}(2 \pi)^{4} g^{\mu \nu} \partial_{\mu} \partial_{\nu} \delta^{4}(p)-\delta^{a b} e_{q} \frac{\langle\bar{q} q\rangle}{192} \\
& \times\left[\sigma \cdot F g^{\mu \nu}-\frac{1}{3} \gamma^{\mu} \sigma \cdot F \gamma^{\nu}\right](2 \pi)^{4} \partial_{\mu} \partial_{\nu} \delta^{4}(p)-\delta^{a b} e_{q} \chi \frac{\langle\bar{q} q\rangle}{24} \sigma \cdot F(2 \pi)^{4} \delta^{4}(p)-\delta^{a b} e_{q} \kappa \frac{\langle\bar{q} q\rangle}{192} \\
& \times\left[\sigma \cdot F g^{\mu \nu}-\frac{1}{3} \gamma^{\mu} \sigma \cdot F \gamma^{\nu}\right](2 \pi)^{4} \partial_{\mu} \partial_{\nu} \delta^{4}(p)+i \delta^{a b} e_{q} \xi \frac{\langle\bar{q} q\rangle}{768} \\
& \times\left[\sigma^{\delta \rho} g^{\mu \nu}-\frac{1}{3} \gamma^{\mu} \sigma^{\delta \rho} \gamma^{\nu}\right] \gamma_{5} \epsilon_{\alpha \beta \delta \rho} F^{\alpha \beta}(2 \pi)^{4} \partial_{\mu} \partial_{\nu} \delta^{4}(p)+\cdots
\end{aligned}
$$

in momentum space with $\hat{p} \equiv p_{\mu} \gamma^{\mu}$. Here we follow [6,7] and do not introduce induced condensates of higher dimensions, while we find that the strange quark mass correction is important so that we have explicitly kept it in our calculation.

Only the condensates with even dimensions contribute to the structure $(\sigma \cdot F \hat{p}+\hat{p} \sigma \cdot F)$. They are $1, \chi\langle 0|\bar{q} q| 0\rangle m_{s}$, $m_{s}\langle 0|\bar{q} q| 0\rangle, \quad\left\langle 0\left|g_{s}^{2} G_{\alpha \beta}^{n} G_{\alpha \beta}^{n}\right| 0\right\rangle, \quad \chi\langle 0|\bar{q} q| 0\rangle^{2}$, $\chi\langle 0|\bar{q} q| 0\rangle\left\langle 0\left|g_{s} \bar{q} \sigma \cdot G q\right| 0\right\rangle, \kappa\langle 0|\bar{q} q| 0\rangle^{2}$, and $\xi\langle 0|\bar{q} q| 0\rangle^{2}$ up to dimension eight. The up and down quark is treated as massless. The gluon condensate $\left\langle 0\left|g_{s}^{2} G_{\alpha \beta}^{n} G_{\alpha \beta}^{n}\right| 0\right\rangle$ always appears with a small numerical factor $1 /(2 \pi)^{4}$ through the two-loop integration [6]. Its contribution was found to be negligible through the direct calculation [9]. Following Refs. $[3,6,7]$ the four quark condensate $\left\langle 0\left|\bar{q} \Gamma_{1} q \bar{q} \Gamma_{2} q\right| 0\right\rangle$ is treated by the factorization approximation as the susceptibilities are estimated under the the vacuum dominance hypothesis. The calculation is straightforward by substituting the 
quark propagator into Eqs. (9) and (14). Here we present the final result after Borel transformation:

$$
\begin{aligned}
& \frac{9}{4} e_{s}\left\{M_{B}^{6} E_{2}\left(y_{1}\right) L^{4 / 27}-\frac{6}{5} \chi m_{s} a_{s} M_{B}^{4} E_{1}\left(y_{1}\right) L^{-4 / 9}\right. \\
& +\frac{6}{5} m_{s} a_{s} M_{B}^{2} E_{0}\left(y_{1}\right) L^{4 / 27}-\frac{4}{5} \chi a_{s}^{2} M_{B}^{2} E_{0}\left(y_{1}\right) L^{4 / 9} \\
& \left.+a_{s}^{2} L^{28 / 27}\left[\frac{14}{15}+\frac{\chi m_{0}^{2}}{30} L^{-10 / 9}-\frac{22}{15} \kappa-\frac{4}{15} \xi\right]\right\} \\
& =(2 \pi)^{4} \lambda_{\Omega}^{2} e^{-m_{\Omega}^{2} / M_{B}^{2}} \mu_{\Omega}\left(1+A_{1} M_{B}^{2}\right), \\
& \frac{1}{\sqrt{3}}\left(e_{u}-e_{d}\right)\left\{M_{B}^{6} E_{2}\left(y_{2}\right) L^{-4 / 9}-\chi m_{s} a M_{B}^{4} E_{1}\left(y_{2}\right) L^{-28 / 27}\right. \\
& +\frac{4}{3} m_{s} a M_{B}^{2} E_{0}\left(y_{2}\right) L^{-4 / 9}-m_{s} a_{s} M_{B}^{2} E_{0}\left(y_{2}\right) L^{-4 / 9} \\
& -\frac{2}{3} \chi a a_{s} M_{B}^{2} E_{0}\left(y_{2}\right) L^{-4 / 27}+\frac{1}{9} a a_{s} L^{4 / 9} \\
& \left.\times\left[4+\kappa-2 \xi+\frac{3}{4} \chi m_{0}^{2} L^{-10 / 9}\right]\right\} \\
& =(2 \pi)^{4} \lambda_{\Sigma} \lambda_{\Lambda} e^{-\vec{m}^{2} / M_{B}^{2}} \mu_{\Sigma^{0} \Lambda}\left(1+A_{2} M_{B}^{2}\right) \text {, }
\end{aligned}
$$

where $m_{\Omega}=1.672 \mathrm{GeV}, \bar{m}=1.15 \mathrm{GeV}, m_{s}=150 \mathrm{MeV}$, $y_{1}=W_{1}^{2} / M_{B}^{2}$ and $y_{2}=W_{2}^{2} / M_{B}^{2} . E_{n}(y)=1-e^{-y} \sum_{k=0}^{n} 1 / k ! y^{k}$ are the factors used to subtract the continuum contribution [6]. $W_{1}^{2}=5.0 \mathrm{GeV}^{2}$ and $W_{2}^{2}=3.4 \mathrm{GeV}^{2}$ are the continuum thresholds which are determined together with the overlap amplititudes $(2 \pi)^{4} \lambda_{\Omega}^{2}=5.56 \mathrm{GeV}^{6},(2 \pi)^{4} \lambda_{\Sigma}^{2}=1.88 \mathrm{GeV}^{6}$, and $(2 \pi)^{4} \lambda_{\Lambda}^{2}=1.64 \mathrm{GeV}^{6}$ from the $\Omega, \Sigma$, and $\Lambda$ mass sum rules $[11,12]$. We adopt the "standard" values for the various condensates $a=-(2 \pi)^{2}\langle 0|\bar{u} u| 0\rangle=0.55$ $\mathrm{GeV}^{3}, \quad a_{s}=-(2 \pi)^{2}\langle 0|\bar{s} s| 0\rangle=0.55 \times 0.8 \quad \mathrm{GeV}^{3}, \quad a m_{0}^{2}$ $=(2 \pi)^{2} g_{s}\langle 0|\bar{u} \sigma \cdot G u| 0\rangle, \quad m_{0}^{2}=0.8 \quad \mathrm{GeV}^{2} . \quad L=\ln \left(M_{B} /\right.$ $\left.\Lambda_{\mathrm{QCD}}\right) / \ln \left(\mu / \Lambda_{\mathrm{QCD}}\right), \Lambda_{\mathrm{QCD}}$ is the QCD parameter, $\Lambda_{\mathrm{QCD}}$ $=100 \mathrm{MeV}$, and $\mu=0.5 \mathrm{GeV}$ is the normalization point to which the used values of condensates are referred.

We further improve the numerical analysis by taking into account of the renormalization group evolutions of the sum rules (17) and (18) through the anomalous dimensions of the various condensates and currents. $A_{1}$ and $A_{2}$ are constants to be determined from the sum rule, which arise from the nondiagonal transitions $\Omega \gamma \rightarrow \Omega^{*}, \Sigma * \rightarrow \Lambda \gamma$, or $\Sigma \rightarrow \Lambda^{*} \gamma$ [6]. The working intervals of the Borel mass $M_{B}^{2}$ for the sum rules (17) and (18) are $2.0 \mathrm{GeV}^{2} \leqslant M_{B}^{2} \leqslant 4.0 \mathrm{GeV}^{2}$ and $1.3 \mathrm{GeV}^{2} \leqslant M_{B}^{2} \leqslant 3.0 \mathrm{GeV}^{2}$, respectively, where both the continuum contribution and power corrections are controllable. Moving the factor $(2 \pi)^{4} \lambda_{\Omega}^{2} e^{-m_{\Omega}^{2} / M_{B}^{2}}$ and $(2 \pi)^{4} \lambda_{\Sigma} \lambda_{\Lambda} e^{-\bar{m}^{2} / M_{B}^{2}}$ on the right-hand side to the left and fitting the new sum rules with a straight line approximation we may extract the $\mu_{\Omega}$ and $\mu_{\Sigma \Lambda}$. We show the new sum rules as a function of the Borel mass in Fig. 1. The new sum rules are almost stable and independent of $M_{B}^{2}$, which implies that the nondiagonal transition contributions are small.

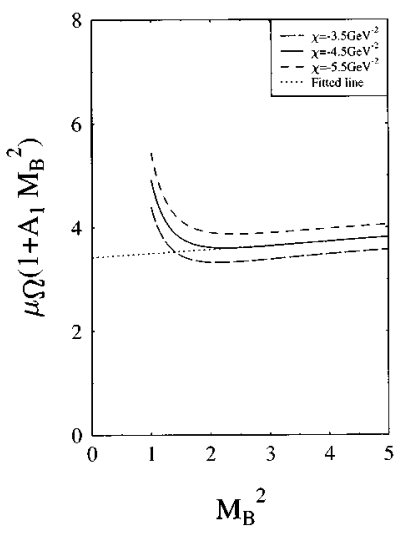

(a)

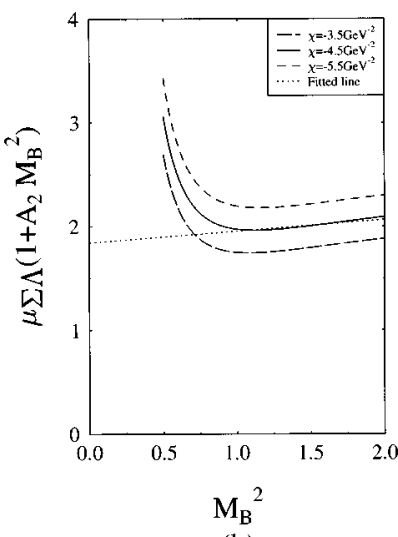

(b)
FIG. 1. (a) The Borel mass dependence of the $\Omega$ magnetic moment. The long-dashed, solid, and short-dashed curve are the QCD sum rule predictions for $\chi=-3.5,-4.5$, and $-5.5 \mathrm{GeV}^{-2}$, respectively, from Eq. (17) after the numerical factor $(2 \pi)^{4} \lambda_{\Omega}^{2} e^{-m_{\Omega}{ }^{2} / M_{B}^{2}}$ is moved to the left-hand side. The dotted line is a straight-line approximation. The intersect with the $Y$ axis is the $\Omega$ magnetic moment in units of $e / 2 m_{\Omega}$. The Borel mass $M_{B}^{2}$ is in units of $\mathrm{GeV}^{2}$. (b) The Borel mass dependence of the $\Sigma \Lambda$ transition magnetic moment. The notations are the same as those in (a).

The sum rules are insensitive to the susceptibilities $\kappa$ and $\xi$ because of their small values. Their contributions are less than $5 \%$. The dependence on $\chi$ is shown in Fig. 1. When $\chi$ varies from -4.5 to $-3.5 \mathrm{GeV}^{-2}$ or to $-5.5 \mathrm{GeV}^{-2}$, the sum rules change within $10 \%$. The correction from the strange quark mass is important and contributes about $20 \%$ to both of the sum rules. The $\mathrm{SU}(3)_{f}$ flavor symmetry breaking parameter $\gamma=\langle\bar{s} s\rangle\langle\langle\bar{u} u\rangle$ needs to take the standard value of 0.8 in order to yield a good agreement with the experimental data in Eq. (17). The $\Omega$ magnetic moment would increase $30 \%$ if $\gamma=1$, in contradiction with the experimental data. Our final results are $\mu_{\Omega}=-3.41$ in units of $e / 2 m_{\Omega}$ and $\mu_{\Sigma 0_{\Lambda}}=1.85$ in units of $e / 2 \bar{m}$, where $e / 2 m_{B}$ is a natural unit in QCD sum rule analyses. In unit of nuclear magneton $\mu_{\Omega}=-1.92 \mu_{N}$ and $\mu_{\Sigma{ }^{0}}=1.5 \mu_{N}$, in good agreement with the recent experimental data.

Baryon magnetic moments are important physical observables as masses. The method of QCD sum rules in the presence of an external electromagnetic field was successfully employed to calculate the octet baryon magnetic moments. The results are in reasonable agreement with the experimental data. In this work we have extended the same method to calculate the magnetic moment of the long-lived decuplet member, the $\Omega$ and $\Sigma^{0} \Lambda$ transition magnetic moments simultaneously, which may serve both as a consistency check of the various susceptibilities and a check of the method of the external field itself. Our results are in good agreement with the recent experimental data.

This work was supported in part by the Postdoctoral Science Foundation of China and the National Natural Science Foundation of China. It was also supported in part by the National Science Council of R.O.C. (Taiwan) under Grant No. NSC84-2112-M002-021Y. 
[1] J. Franklin, Phys. Rev. D 29, 2648 (1984).

[2] Y. Tomowaza, Phys. Rev. D 19, 1626 (1979); T. Das and S. P. Misra, Phys. Lett. 96B, 165 (1980); C. Bernard et al., Phys. Rev. Lett. 49, 1076 (1982); H. J. Lipkin, Nucl. Phys. B214, 136 (1982); H. Georgi and A. Monohar, Phys. Lett. 132B, 183 (1983); V. P. Efrosinin and D. A. Zaikin, Yad. Fiz. 44, 1053 (1986) [Sov. J. Nucl. Phys. 44, 681 (1986)]; R. C. Verma and M. P. Khanna, Phys. Lett. B 183, 207 (1987); L. Brekke and J. L. Rosner, Comments Nucl. Part. Phys. 18, 83 (1988); M. Krivoruchenko et al., Phys. Rev. D 41, 997 (1990); J. Kunz and P. J. Mulders, ibid. 41, 1578 (1990).

[3] M. A. Shifman, A. I. Vaishtein, and V. I. Zakharov, Nucl. Phys. B147, 385 (1979); B147, 448 (1979).

[4] N. B. Wallace et al., Phys. Rev. Lett. 74, 3732 (1995).
[5] Particle Data Group, R. M. Barnett et al., Phys. Rev. D 54, 1 (1996), p. 55.

[6] B. L. Ioffe and A. V. Smilga, Nucl. Phys. B232, 109 (1984).

[7] I. I. Balitsky and A. V. Yung, Phys. Lett. 129B, 328 (1983).

[8] V. M. Belyaev and Ya. I. Kogan, Yad. Fiz. 40, 1035 (1984) [Sov. J. Nucl. Phys. 40, 659 (1984)].

[9] C. B. Chiu, J. Pasupathy, and S. J. Wilson, Phys. Rev. D 33, 1961 (1986); S. L. Wilson, I. Pasupathy, and C. B. Chiu, ibid. 36, 1451 (1987); C. B. Chiu, S. L. Wilson, J. Pasupathy, and J. P. Singh, ibid. 36, 1553 (1987).

[10] Y. Takhashi, An Introduction to Field Quantization (Pergamon, New York, 1969).

[11] L. J. Reinders, H. Rubinstein, and S. Yazaki, Phys. Lett. 120B, 209 (1983).

[12] W-Y. Hwang and K-C. Yang, Phys. Rev. D 49, 460 (1994). 